\title{
ODD HARMONIOUS LABELINGS OF CYCLIC SNAKES
}

\author{
M. E. Abdel-Aal \\ Department of Mathematics, Faculty of Science, \\ Benha University, Benha 13518, Egypt
}

\begin{abstract}
In [8] Liang and Bai have shown that the $k C_{4}$ - snake graph is an odd harmonious graph for each $k \geq 1$. In this paper we generalize this result on cycles by showing that the $k C_{n}$ - snake with string $1,1, \ldots, 1$ when $n \equiv 0(\bmod 4)$ are odd harmonious graph. Also we show that the $k C_{4}-$ snake with m-pendant edges for each $k, m \geq 1$, (for linear case and for general case). Moreover, we show that, all subdivision of $2 m \Delta_{k}-$ snake are odd harmonious for each $k, m \geq 1$. Finally we present some examples to illustrate the proposed theories.
\end{abstract}

\section{KEYWORDS}

Odd harmonious labeling, pendant edges, Cyclic snakes, Subdivision double triangular snakes.

\section{INTRODUCTION}

Graph labeling is an active area of research in graph theory which has mainly evolved through its many applications in coding theory, communication networks, mobile telecommunication system. Optimal circuits layouts or graph decompositions problems, no name just a few of them.

Most graph labeling methods trace their origion to one introduced by Rosa [1] called such a labeling a $\beta$-valuation and Golomb [2] subsequently called graceful labeling, and one introduced by Graham and Sloane [3] called harmonious labeling. Several infinite families of graceful and harmonious graphs have been reported. Many illustrious works on graceful graphs brought a tide to different ways of labeling the elements of graph such as odd graceful.

A graph $G$ of size $q$ is $o d d$-graceful, if there is an injection $f$ from $\mathrm{V}(G)$ to $\{0,1,2, \ldots, 2 q-1\}$ such that, when each edge $x y$ is assigned the label or weight $|f(x)-f(y)|$, the resulting edge labels are $\{1,3,5, \ldots, 2 q-1\}$. This definition was introduced by Gnanajothi [4]. Many researchers have studied odd graceful labeling. Seoud and Abdel-Aal [5] they determine all connected odd graceful graphs of order $\leq 6$. For a dynamic survey of various graph labeling problems we refer to Gallian [6].

DOI : 10.5121/jgraphoc.2013.5301 
International journal on applications of graph theory in wireless ad hoc networks and sensor networks (GRAPH-HOC) Vol.5, No.3, September 2013

Throughout this work graph $\mathrm{G}=(\mathrm{V}(\mathrm{G}), \mathrm{E}(\mathrm{G}))$ we mean a simple, finite, connected and undirected graph with $p$ vertices and $q$ edges. For all other standard terminology and notions we follow Harary [7].

A graph $\mathrm{G}$ is said to be odd harmonious if there exists an injection $f: V(G) \rightarrow\{0,1,2, \ldots, 2 q-1\}$ such that the induced function $f^{*}: E(G) \rightarrow\{1,3, \ldots, 2 q-1\}$ defined by $f^{*}(u v)=f(u)+f(v)$ is a bijection. Then $f$ is said to be an odd harmonious labeling of $G$ [8].

A graph $k C_{n}$-snake was introduced by Barrientos [9], as generalization of the concept of triangular snake introduced by Rosa [10].

Let $G$ be a $k C_{n}-$ snake with $k \geq 2$. Let $u_{1}, u_{2}, \ldots, u_{k-1}$ be the consecutive cut-vertices of $G$. Let $d_{i}$ be the distance between $u_{i}$ and $u_{i+1}$ in $G, 1 \leq i \leq k-2$ and the string $\left(d_{1}, d_{2}, \ldots, d_{k-2}\right)$ of integers. Hence, any graph $G=k C_{n}-$ snake, can be represented by a string. For instance, the string (from left to right) of the $8 C_{4}$ - snake on Figure (4) is 2,2,1,2,1,1. Gracefulness of the kind of $k C_{4}$-snake studied by Gnanajothi have string $1,1, \ldots, 1$. And the labelings given by Ruiz considered by $k C_{4}$-snake with string $2,2, \ldots, 2$. We obtain in Theorem 2.4 an odd harmonious labelings of the $k C_{4}$ - snake with string $1,1, \ldots, 1$. If the string of given $k C_{n}-$ snake is $\left\lfloor\frac{n}{2}\right\rfloor,\left\lfloor\frac{n}{2}\right\rfloor, \ldots,\left\lfloor\frac{n}{2}\right\rfloor$, we say that $k C_{n}-$ snake is linear.

This paper can be divided into two sections. Section 1 , we show that the graphs $k C_{4}-$ snake $m k_{1}$ (the graph obtained by joining $m$-pendant edges to each vertex of $k C_{4}-$ snake $k, m \geq 1$ ) for linear and general cases of $k C_{4}-$ snake for each $k \geq 1$ are odd harmonious. We also obtain an odd harmonious labeling of $k C_{n}$-snake with the sequence string is $1,1, \ldots, 1$ and when $n \equiv 0(\bmod 4)$. In section 2 , we show that, an odd harmonious labeling of the all subdivision of double triangular snakes $\left(2 \Delta_{k}-\right.$ snake). Finally, we prove that the all subdivision of $2 m \Delta_{k}$ snake are odd harmonious for each $k, m \geq 1$.

\section{Main Results}

In [8, Corollary 3.2 ] Liang and Bai in Corollary 3.2 (2) when $\mathrm{i}=1$, they have shown that, the $k C_{4}$-snake graph is an odd harmonious graph for each $k \geq 1$. We extended this result to obtain an odd harmonious labeling for the corona $k C_{4}$ - snake graph (the graph obtained by joining $\mathrm{m}$ pendant edges to each vertex $k C_{4}$-snake, ) are denoted by $k C_{4}$-snake $m k_{1}$.

Theorem 2.1.

The linear graphs $k C_{4}$-snake $\quad m k_{1}$ are odd harmonious for $k, m \geq 1$ 
International journal on applications of graph theory in wireless ad hoc networks and sensor networks (GRAPH-HOC) Vol.5, No.3, September 2013

Proof. Consider the linear graph $k C_{4}$-snake, $k \geq 1$ which has the vertices $w_{i}, u_{j}$, and $v_{j}$ where $i=0,1,2, \ldots, k, j=1,2, \ldots, k$. In order to get the linear $k C_{4}-$ snake $m k_{1}, k, m \geq 1$, we add m-pendant edges $w_{i}^{l}, u_{j}^{l}$, and $v_{j}^{l}$ to each vertex of $w_{i}, u_{j}$, and $v_{j}$ respectively such that $l=0,1,2, \ldots, m$. Now, let $\mathrm{G}$ be the linear $k C_{4}$-snake $m k_{1}, k, m \geq 1$ be described as indicated in Figure 1.

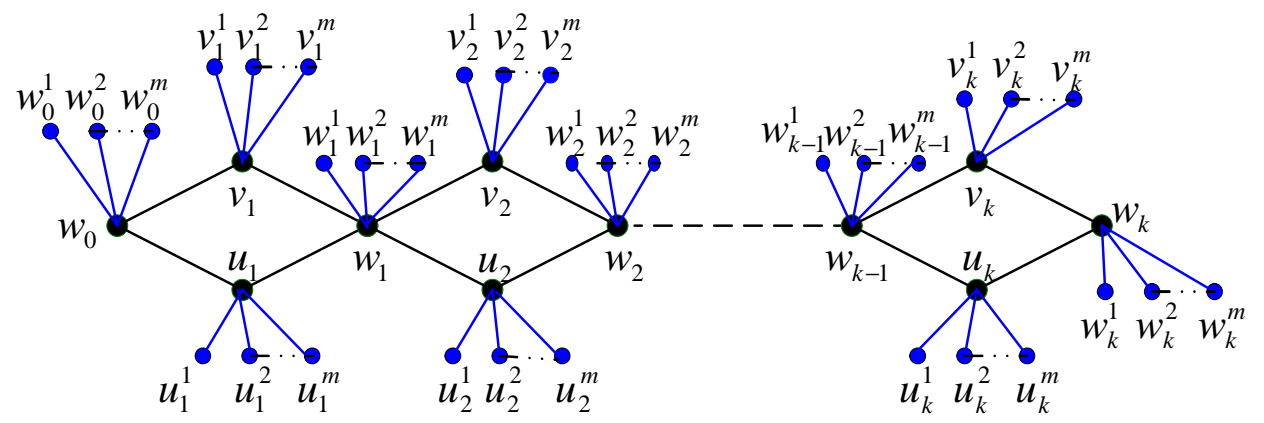

Figure 1

It is clear that the number of edges of the graph $H$ is $q=4 k+m(3 k+1)$. We define the labeling function $f: V(G) \rightarrow\{0,1,2, \ldots, 8 k+2 m(3 k+1)-1\}$ as follows:

$$
\begin{aligned}
& f\left(w_{i}\right)=4 i, \quad 0 \leq i \leq k . \\
& f\left(v_{i}\right)=4 i-3, \quad 1 \leq i \leq k, \\
& f\left(u_{i}\right)=4 i-1, \quad 1 \leq i \leq k, \\
& f\left(w_{i}^{j}\right)=2[q-j-(m+2) i]+1, \quad 0 \leq i \leq k, \quad 1 \leq j \leq m \\
& f\left(u_{i}^{j}\right)=4 k(m+2)-2 j-4(m+1) i+2 m+2, \quad 1 \leq i \leq k, \quad 1 \leq j \leq m \\
& f\left(v_{i}^{j}\right)=4 k(m+2)-2 j-4(m+1)(i-1), \quad 1 \leq i \leq k, \quad 1 \leq j \leq m .
\end{aligned}
$$

The edge labels will be as follows:

- The vertices $w_{i-1}, v_{i}, 1 \leq i \leq k$, induce the edge labels

$$
f\left(w_{i-1}\right)+f\left(v_{i}\right)=8 i-7,1 \leq i \leq k .
$$

- The vertices $w_{i-1}, u_{i}, 1 \leq i \leq k$, induce the edge labels

$$
f\left(w_{i-1}\right)+f\left(u_{i}\right)=8 i-5,1 \leq i \leq k .
$$

- The vertices $v_{i}, w_{i} 1 \leq i \leq k$, induce the edge labels

$$
f\left(v_{i}\right)+f\left(w_{i}\right)=8 i-3,1 \leq i \leq k .
$$

- The vertices $u_{i}, w_{i} 1 \leq i \leq k$, induce the edge labels

$$
f\left(u_{i}\right)+f\left(w_{i}\right)=8 i-1,1 \leq i \leq k .
$$

The remaining odd edge labels from $8 k+1$ to $2 k+2 m(3 k+1)$ are obtained from the following

- $f\left(u_{j}\right)+f\left(u_{j}^{j}\right)=4 k(m+2)-2 j-4 m i+2 m+1, \quad 1 \leq i \leq k, \quad 1 \leq j \leq m$.

- $f\left(v_{j}\right)+f\left(v_{j}^{j}\right)=4 k(m+2)-2 j-4 m i+4 m+1, \quad 1 \leq i \leq k, \quad 1 \leq j \leq m$, 
International journal on applications of graph theory in wireless ad hoc networks and sensor networks (GRAPH-HOC) Vol.5, No.3, September 2013

- $f\left(w_{i}\right)+f\left(w_{i}^{j}\right)=2[q-j-m i]+1, \quad 0 \leq i \leq k, \quad 1 \leq j \leq m$, So $\{f(u)+f(v)$ : $u v \in E(G)\}=\{1,3,5, \ldots, 2 q-1\}$. Hence the graph $G$ is odd harmonious.

Example 2.3. An odd harmonious labeling of the graph linear $3 C_{4}-$ snake $2 k_{1}$, is shown in Figure (2).

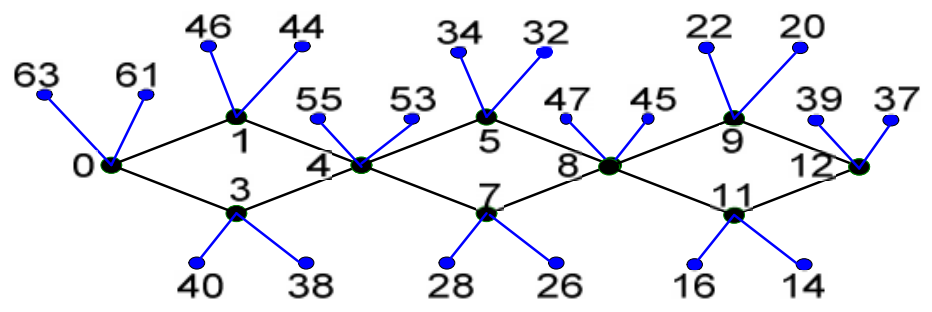

Figure (2): The graph linear $3 C_{4}-$ snake $2 k_{1}$ with its odd harmonious labeling.

For the general form of cyclic graph we obtain the following result.

Theorem 2.2.

The following graphs are odd harmonious

(i) $\quad k C_{4}-$ snake for each $k \geq 1$,

(ii) $\quad k C_{4}-$ snake $\quad m k_{1}$ for each $k, m \geq 1$,(the general form).

Proof.

The graph $k C_{4}-$ snake can be considered as a bipartite graph (one partite set has black vertices and the other has white vertices) it is possible to embed it, on a square grid as is showed in the next Figure 3.

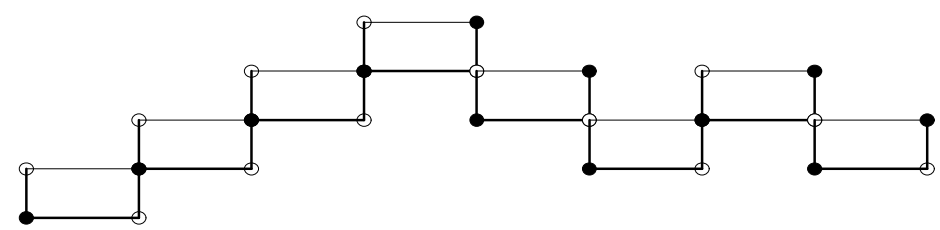

Figure 3

Let $\mathrm{G}$ be the graph $k C_{4}$-snake $m k_{1}$ which obtained by joining $m$ - pendant edges to each vertex of $k C_{4}-$ snake. Then $|V(G)|=(m+1)(3 k+1)$ and $|E(G)|=4 k+m(3 k+1)$. Now, we are running the following steps sequentially in order to prove the Theorem:

Step 1. Since $k C_{4}-$ snake is a bipartite graph so it has one partite set has black vertices and the other has white vertices as is showed in Figure (3). Put black vertices in a string, ordered by diagonals from left to right and inside each diagonal from bottom to top, assign to them from an arithmetic progression of difference 4 which first term is zero, when we move to another diagonal 
International journal on applications of graph theory in wireless ad hoc networks and sensor networks (GRAPH-HOC) Vol.5, No.3, September 2013

we use an arithmetic progression of difference 2, counting until the last black vertex has been numbered. Similarly, Put the white vertices on a string, ordered for diagonals from left to right and inside each diagonal from top to bottom, starting with the first diagonal assign numbers from an arithmetic progression of difference 2, which first term is 1 , when we move to another diagonal we use an arithmetic progression of difference 2, counting until the last white vertex has been numbered.

Step 2. In this step, we are labeling the vertices of $m$-pendant edges which contact with the white diagonals, from right to left and inside each white diagonal from bottom to top, assign to them from an arithmetic progression of difference 2, which first term is $z$ such that $z=y+2$ where $y$ is the last vertex labeling of black diagonal, when we move to a new vertex of the white diagonal, the first vertex of $m$ - pendant edges is labeled by an arithmetic progression of difference 4 , but the arithmetic progression of difference 2 has been used with the remain $(m-1)$ vertices of $m$-pendant edges. We move from a vertex to another of the white diagonals until the last white vertex.

Step 3. Finally, we are labeling the vertices of $m$-pendant edges which contact with the black diagonals, from left to right and inside each black diagonal from bottom to top, assign to them from an arithmetic progression of difference (-2), which first term is $(2 q-1)$ where $q$ is the size of $G$, when to move to a new vertex of the black diagonal, the first vertex of $m$-pendant edges is labeled by an arithmetic progression of difference (-6), but the arithmetic progression of difference( -2$)$ has been used with the remain $(m-1)$ vertices of $m$-pendant edges. We move from a vertex to another of the black diagonals until the last black vertex.

Now, we have complete the proof by running the above steps, i.e. we mention only the vertices labels and the reader can fulfill the proof as we did in the previous theorem where step 1 give us an odd harmonious labeling of the graph $k C_{4}$ - snake for each $k \geq 1$, and step1- step3 give us an odd harmonious labeling of the graph $k C_{4}$-snake $m k_{1}$ for each $k, m \geq 1$ (in general case).

The following example illustrates the last result.

Example 2.3. An odd harmonious labeling of the graph $5 C_{4}-$ snake $2 k_{1}$ (for general case) is shown in Figure 4.

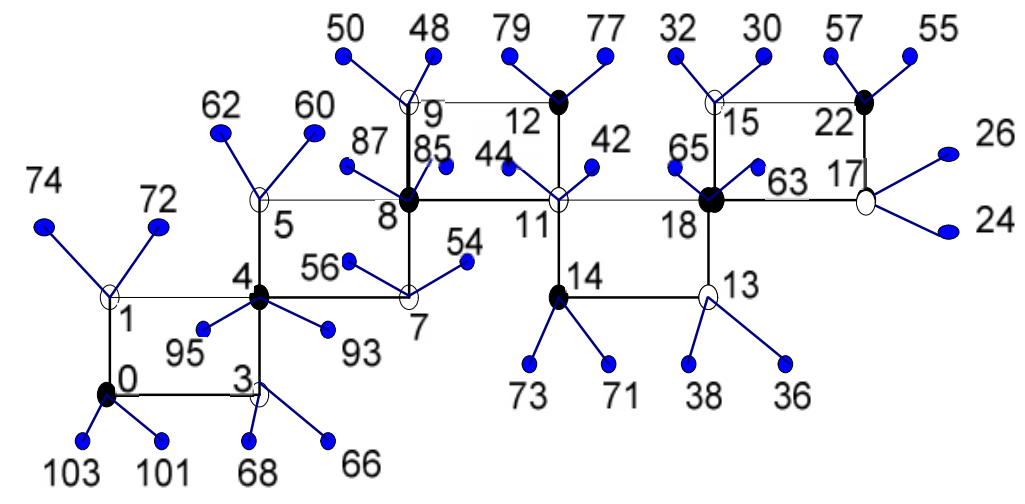

Figure 4: The graph $5 C_{4}$-snake $2 k_{1}$ (for general case) with its odd harmonious labeling. 
International journal on applications of graph theory in wireless ad hoc networks and sensor networks (GRAPH-HOC) Vol.5, No.3, September 2013

The graphs $k C_{n}-$ snake when the sequence string is $(1,1,1, \ldots, 1)$ when $n \equiv 0(\bmod 4)$ are studied in the following Theorem:

Theorem 2.4. The graphs $k C_{4 m}$-snake for each $k, m \geq 1$, with string $(1,1, \ldots, 1)$ are odd harmonious.

Proof. Let $G=k C_{4 m}-$ snake $=(n-1) C_{4 m}-$ snake can be described as indicated in Figure 5

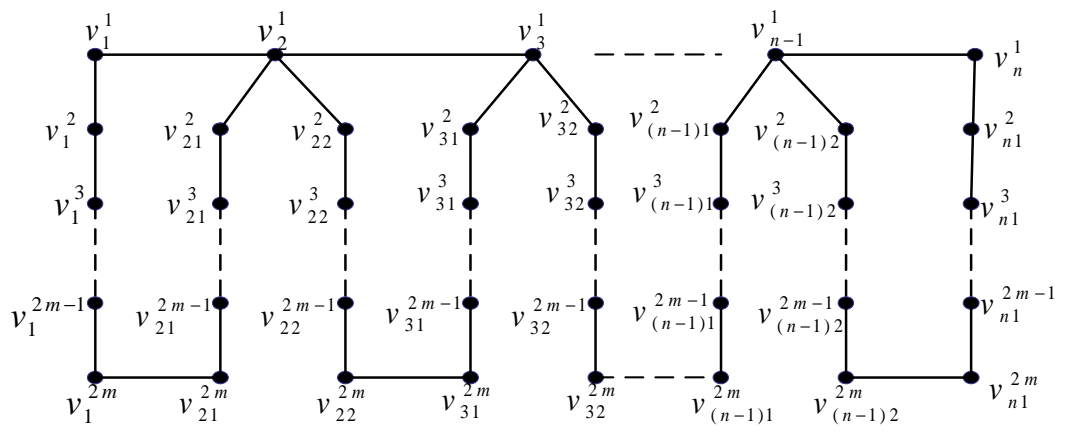

Figure 5

It is clear that $|E(G)|=4 m(n-1)$. We define the labeling function

as follows:

$$
f: V(G)) \rightarrow\{0,1,2, \ldots, 8 m(n-1)-1\}
$$

$$
\begin{aligned}
& f\left(v_{i}^{1}\right)=\left\{\begin{array}{l}
4 m(i-1), \quad i=1,3,5, \ldots n \text { or } n-1 \\
4 m(i-1)+1, \quad i=2,4,6, \ldots n-1 \text { or } n .
\end{array}\right. \\
& f\left(v_{1}^{j}\right)=j-1, \quad 1 \leq j \leq 2 m
\end{aligned}
$$

For labeling the vertices $v_{i 1}^{j}, 2 \leq i \leq n, 2 \leq j \leq 2 m$ we consider the following two cases:

\section{Case $(i)$}

if $i$ is odd, $3 \leq i \leq n$ we have the following labeling, for each $2 \leq j \leq 2 m$

$$
f\left(v_{i 1}^{j}\right)=f\left(v_{i}^{1}\right)-j+1
$$

\section{Case(ii)}

if $i$ is even, $2 \leq i \leq n$ we have the following labeling, for each $2 \leq j \leq 2 m$ :

$$
f\left(v_{i 1}^{j}\right)= \begin{cases}f\left(v_{i}^{1}\right)-j+1, & j=1,3,5, \ldots 2 m-1 \\ f\left(v_{i}^{1}\right)-j-1, & j=2,4,6, . .2 m .\end{cases}
$$

Now we label the remaining vertices $v_{i 2}^{j}, \quad 2 \leq i \leq n-1,1 \leq j \leq 2 m$ as follows:

$$
f\left(v_{i 2}^{j}\right)=f\left(v_{i 1}^{1}\right)+2(j-1), 1 \leq j \leq 2 m, 2 \leq i \leq n-1 .
$$


International journal on applications of graph theory in wireless ad hoc networks and sensor networks (GRAPH-HOC) Vol.5, No.3, September 2013

It follows that $f$ admits an odd harmonious labeling for $(n-1) C_{4 m}-$ snake. Hence $(n-1) C_{4 m}-$ snake for each $n \geq 2, m \geq 1$ with the string $(1,1, \ldots, 1)$ are odd harmonious graphs.

Example 2.5. Odd harmonious labeling of graph $4 C_{8}$ - snake with the string $(1,1, \ldots, 1)$ is shown in Figure 6.

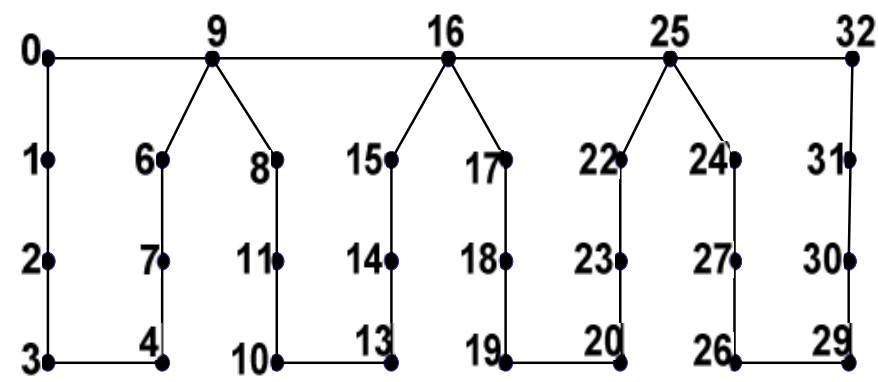

Figure 6: The graph $4 C_{8}-$ snake with its odd harmonious labeling.

\section{Subdivision OF Double Triangles Snake}

Rosa [10] defined a triangular snake (or $\Delta$-snake) as a connected graph in which all blocks are triangles and the block-cut-point graph is a path. Let $\Delta_{k}$-snake be a $\Delta$-snake with $k$ blocks while $n \Delta_{k}$-snake be a $\Delta$-snake with $k$ blocks and every block has $n$ number of triangles with one common edge.

David Moulton [11] proved that triangular snakes with $\mathrm{p}$ triangles are graceful if $\mathrm{p}$ is congruent to 0 or 1 modulo 4 . $\mathrm{Xu}$ [12] proved that they are harmonious if and only if $\mathrm{p}$ is congruent to 0,1 or 3 modulo 4 .

A double triangular snake is a graph that formed by two triangular snakes have a common path. The harmonious labeling of double triangle snake introduced by Xi Yue et al [13]. It is known that, the graphs which contain odd cycles are not odd harmonious so we used the subdivision notation for odd cycle in order to prove that all subdivision of double triangular snakes are odd harmonious.

Theorem 3.1. All subdivision of double triangular snakes $\left(2 \Delta_{k}\right.$-snake $\left.k \geq 1\right)$ are odd harmonious.

Proof. Let $G=2 \Delta_{k}$-snake has $q$ edges and $p$ vertices. The graph $G$ consists of the vertices $\left(u_{1}, u_{2}, \ldots, u_{k+1}\right),\left(v_{1}, v_{2}, \ldots, v_{k}\right),\left(w_{1}, w_{2}, \ldots, w_{k}\right)$ therefore we get the subdivision of double triangular snakes $S(G)$ by subdividing every edge of double triangular snakes $2 \Delta_{k}$-snake exactly once. Let $y_{i}$ be the newly added vertex between $u_{i}$ and $u_{i+1}$ while $w_{i 1}$ and $w_{i 2}$ are newly added vertices between

$w_{i} u_{i}$ and $w_{i} u_{i+1}$ respectively, where $1 \leq i \leq k$. Finally, $v_{i 1}$ and $v_{i 2}$ are newly added vertices between 
International journal on applications of graph theory in wireless ad hoc networks and sensor networks (GRAPH-HOC) Vol.5, No.3, September 2013

$v_{i} u_{i}$ and $v_{i} u_{i+1}$ respectivley, such that $1 \leq i \leq k$. Let the graph $S(G)$ be described as indicated in Figure 7

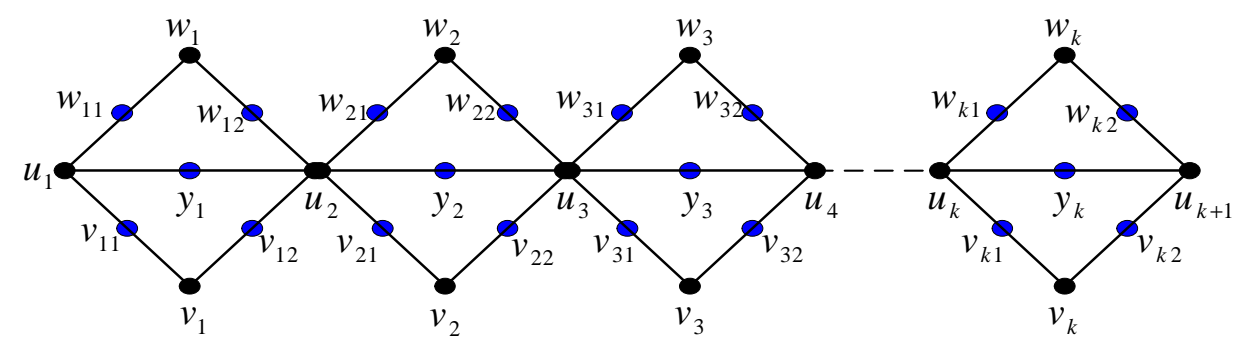

Figure 7: the subdivision of double triangular snakes $\left(2 \Delta_{k}-\right.$ snake).

It is clear that the number of edges of the graph $S(G)$ is $10 \mathrm{k}$. We define the labeling function: as follows:

$$
f: V(S(G)) \rightarrow\{0,1,2,3, \ldots, 20 k-1\}
$$

$$
\begin{aligned}
& f\left(u_{i}\right)=6(i-1), \quad 1 \leq i \leq k+1=n, \\
& f\left(y_{i}\right)=14 i-11, \quad 1 \leq i \leq k=n-1, \\
& f\left(w_{i}\right)=6 i+4, \quad 1 \leq i \leq k=n-1, \\
& f\left(v_{i}\right)=6 i-4, \quad 1 \leq i \leq k=n-1, \\
& f\left(w_{i j}\right)=14 i+8 j-21, \quad 1 \leq i \leq k=n-1, \quad j=1,2, \\
& f\left(v_{i j}\right)=14 i+6 j-15, \quad 1 \leq i \leq k=n-1, \quad j=1,2 .
\end{aligned}
$$

$\underline{\text { The edge labels will be as follows: }}$

- The vertices $u_{i}$ and $w_{i 1}, 1 \leq i \leq k$, induce the edge labels $\{20 i-19,1 \leq i \leq k\}=\{1,21, \ldots, 20 k-19\}$.

- The vertices $u_{i}$ and $y_{i}, 1 \leq i \leq k$, induce the edge labels $\{20 i-17,1 \leq i \leq k\}=\{3,23, \ldots, 20 k-17\}$.

- The vertices $u_{i}$ and $v_{i 1}, 1 \leq i \leq k$, induce the edge labels $\{20 i-15,1 \leq i \leq k\}=\{5,25, \ldots, 20 k-15\}$.

- The vertices $v_{i 1}$ and $v_{i}, 1 \leq i \leq k$, induce the edge labels $\{20 i-13,1 \leq i \leq k\}=\{7,27, \ldots, 20 k-13\}$.

- The vertices $y_{i}$ and $u_{i+1}, 1 \leq i \leq k$, induce the edge labels $\{20 i-11,1 \leq i \leq k\}=\{9,29, \ldots, 20 k-11\}$.

- The vertices $w_{i 1}$ and $w_{i}, 1 \leq i \leq k$, induce the edge labels $\{20 i-9,1 \leq i \leq k\}=\{11,31, \ldots, 20 k-9\}$.

- The vertices $v_{i}$ and $v_{i 2}, 1 \leq i \leq k$, induce the edge labels $\{20 i-7,1 \leq i \leq k\}=\{13,33, \ldots, 20 k-7\}$.

- The vertices $w_{i 2}$ and $u_{i+1}, 1 \leq i \leq k$, induce the edge labels $\{20 i-5,1 \leq i \leq k\}=\{15,35, \ldots, 20 k-5\}$.

- The vertices $v_{i 2}$ and $u_{i+1}, 1 \leq i \leq k$, induce the edge labels $\{20 i-3,1 \leq i \leq k\}=\{17,37, \ldots, 20 k-3\}$.

- The vertices $w_{i}$ and $w_{i 2}, 1 \leq i \leq k$, induce the edge labels $\{20 i-1,1 \leq i \leq k\}=\{19,39, \ldots, 20 k-1\}$.

So we obtain all the edge labels $\{1,3,5, \ldots, 20 k-1\}$. Hence the subdivision of double triangular snakes $\left(2 \Delta_{k}\right.$-snake, $\left.k \geq 1\right)$ are odd harmonious.

Theorem 3.2.

All subdivision of $2 m \Delta_{k}$-snake, $m, k \geq 1$ are odd harmonious. 
International journal on applications of graph theory in wireless ad hoc networks and sensor networks (GRAPH-HOC) Vol.5, No.3, September 2013

Proof. Let $\mathrm{G}=2 m \Delta_{k}$-snake has q edges and $\mathrm{p}$ vertices. The graph $\mathrm{G}$ consists of the vertices ( $\left.\mathrm{u}_{1}, \mathrm{u}_{2}, \ldots, \mathrm{u}_{\mathrm{k}+1}\right),\left(v_{i}^{1}, v_{i}^{2}, \ldots, v_{i}^{m}\right),\left(w_{i}^{1}, w_{i}^{2}, \ldots, w_{i}^{m}\right), 1 \leq i \leq k$. Therefore we get generalized the subdivision of double triangular snakes $\mathrm{S}(\mathrm{G})$ by subdividing every edge of $2 m \Delta_{k}$-snake exactly once. Let $\mathrm{y}_{1}$ be the newly added vertex between $u_{i}$ and $u_{i+1}$ while $w_{i 1}^{j}$ and $w_{i 2}^{j}$ are newly added vertices between $\mathrm{u}_{\mathrm{i}} w_{i}^{j}$ and $w_{i}^{j} u_{i+1}$ respectively. Finally, $v_{i 1}^{j}$ and $v_{i 2}^{j}$ are newly added vertices between $\mathrm{u}_{\mathrm{i}} v_{i}^{j}$ and $v_{i}^{j} \mathrm{u}_{\mathrm{i}+1}$ respectively where $\mathrm{i}=1,2, \ldots, \mathrm{k}$ and $\mathrm{j}=1,2,3, \ldots, \mathrm{m}$ ( Figure 8 ). It is Clear that, the number of edges of the graph $\mathrm{S}(\mathrm{G})$ is $\mathrm{q}=\mathrm{k}(8 \mathrm{~m}+2)$ edges.

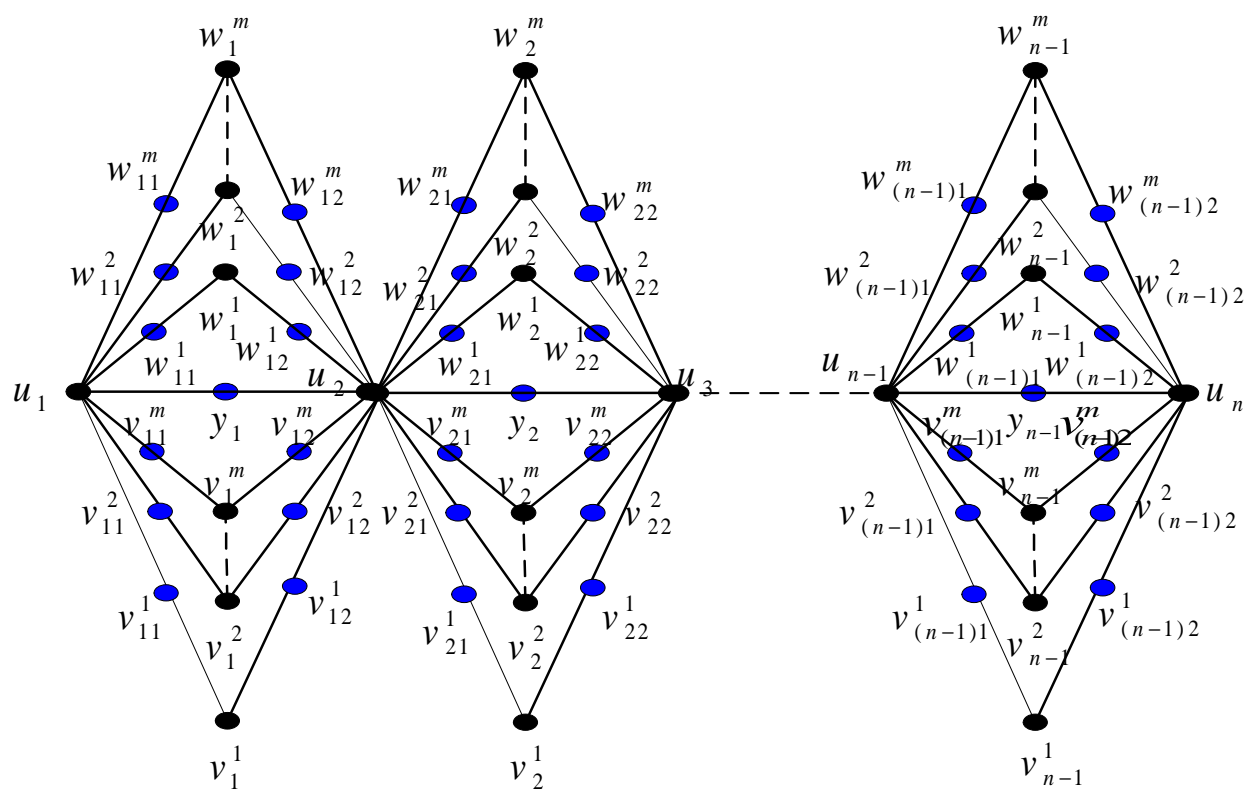

Figure 8: the subdivision of $2 m \Delta_{k}$-snake

We define the labeling function $f: V(S(G)) \rightarrow\{0,1,2,3, \ldots, 2 k(8 m+2)-1\}$ as follows:

$$
\begin{aligned}
& f\left(u_{i}\right)=(4 m+2)(i-1), \quad 1 \leq i \leq k+1=n, \\
& f\left(y_{i}\right)=(12 m+2) i-10 m-1, \quad 1 \leq i \leq k, \\
& f\left(v_{i}^{l}\right)=(4 m+2)(i-1)+4 l-2, \quad 1 \leq i \leq k, 1 \leq l \leq m, \\
& f\left(w_{i}^{j}\right)=(4 m+6)+(4 m+2)(i-1)+4(j-1), \quad 1 \leq i \leq k, 1 \leq j \leq m, \\
& f\left(w_{i l}^{j}\right)=(2 m-1)+(12 m+2)(i-1)+(4 m+2)(l-1)-2(j-1), 1 \leq i \leq k, 1 \leq l \leq 2,1 \leq j \leq m, \\
& f\left(v_{i l}^{j}\right)=(4 m+1)+(12 m+2)(i-1)+(8 m+2)(l-1)-2(j-1), \quad 1 \leq i \leq k, 1 \leq l \leq 2,1 \leq j \\
& f\left(v_{i l}^{1}\right)=(4 m+1)+(12 m+2)(i-1)+(6 m+2)(l-1), \quad 1 \leq i \leq k, \quad 1 \leq l \leq 2 .
\end{aligned}
$$$$
\leq m,
$$ 
International journal on applications of graph theory in wireless ad hoc networks and sensor networks (GRAPH-HOC) Vol.5, No.3, September 2013

In a view of the above defined labeling pattern $f$ is odd harmonious for the graph $S(G)$. Hence $S($ $2 m \Delta_{k}$-snake) is odd-graceful for all $m \geq 1, k \geq 1$.

Illustration 3.3. An odd harmonious labeling of the graph subdivision of $6 \Delta_{3}$-snake is shown in Figure 9.

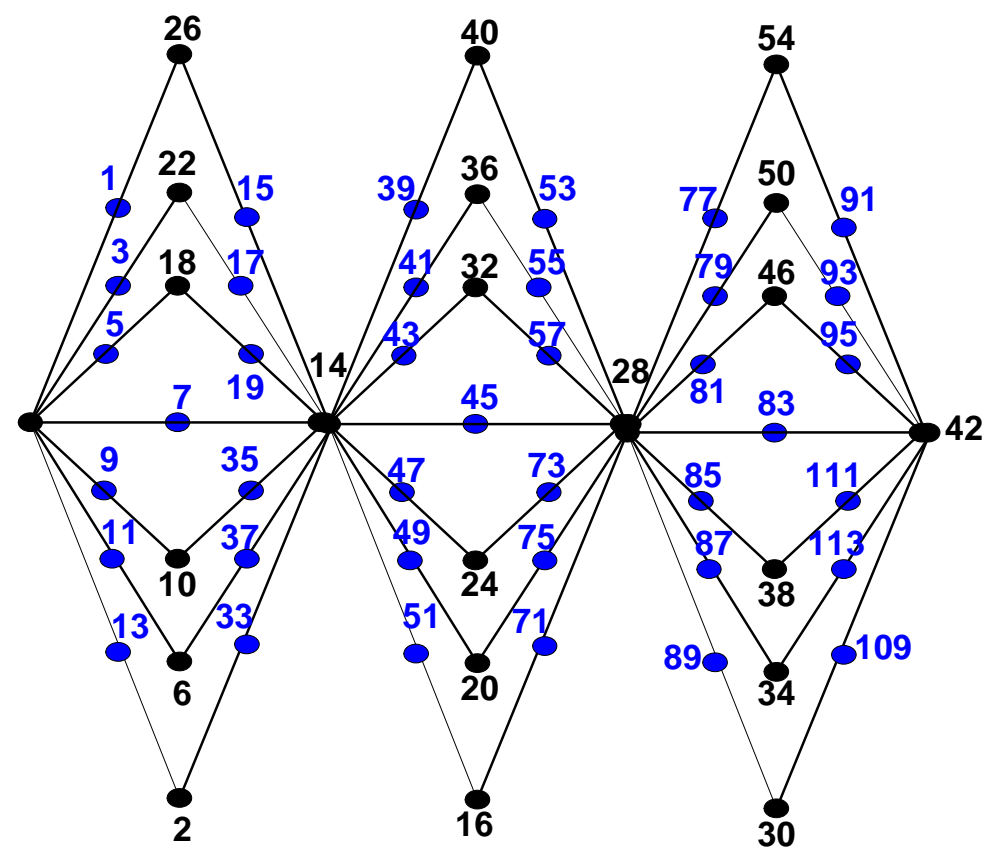

Figure 9: the graph subdivision of $6 \Delta_{3}$-snake with its odd harmonious labeling.

\section{Conclusion}

Harmonious and odd harmonious of a graph are two entirely different concepts. A graph may posses one or both of these or neither. In the present work we investigate several families of odd harmonious cyclic snakes. To investigate similar results for other graph families and in the context of different labeling techniques is open area of research.

\section{REFERENCES}

[1] A. Rosa, (1967) "On certain valuations of the vertices of a graph, in Theory of Graphs", International Symposium, Rome, July 1966, Gordon and Breach, NewYork and Dunod, Paris, pp. 349-355.

[2] S.W. Golomb, (1972) "How to number a graph, in Graph Theory and Computing", R.C. Read, ed.,Academic Press, NewYork, pp. 23-37.

[3] R. L. Graham, N. J. A. Sloane, (1980) "On additive bases and harmonious graphs", SIAM J. Algebr. Disc. Math., Vol 1, No 4, pp. 382-404.

[4] R.B. Gnanajothi, (1991) "Topics in graph theory", Ph.D. thesis, Madurai Kamaraj University, India.

[5] M.A. Seoud and M.E. Abdel-Aal, (2013) On odd graceful graphs, Ars Combin., 108, pp. 161-185.

[6] J. A. Gallian. (2011) “A Dynamic Survey of Graph Labeling”, Electronic J. Combin. Fourteenth edition.

[7] F. Harary. (1969) “GpaphTheory”, Addison-Wesley, Reading MA. 
International journal on applications of graph theory in wireless ad hoc networks and sensor networks

(GRAPH-HOC) Vol.5, No.3, September 2013

[8] Z. Liang, Z . Bai. (2009) On the odd harmonious graphs with applications, J. Appl.Math. Comput., 29, pp.105-116.

[9] C. Barrientos, (2004) "Difference Vertex Labelings", Ph.D.Thesis, University Politecnica De Cataunya, Spain.

[10] A. Rosa, (1967) "Cyclic Steiner Triple System and Labelings of Triangular Cacti”, Scientia, 5, pp. 87-95.

[11] D. Moulton, (1989) "Graceful labelings of triangular snakes", Ars Combin., 28, pp. 3-13.

[12] S.D. Xu, (1995) “ Harmonicity of triangular snakes”, J. Math. Res. Exposition, 15, pp. 475-476

[13] Xi Yue, Yang Yuansheng and Wang Liping, (2008) "On harmonious labeling of the double triangular n snake”, Indian J. pure apple. Math.,39(2), pp. 177-184.

\section{Author}

Mohamed Elsayed Abdel-Aal received the B.Sc. (Mathematics) the M.Sc.(Pure Mathematics-Abstract Algebra) degree from Benha University, Benha, Egypt in 1999, 2005 respectively. Also, he received Ph.D. (Pure Mathematics) degree from Faculty of Mathematics, Tajik National University, Tajikistan, in 2011. He is a University lecturer of Pure Mathematics with the Benha University, Faculty of Science, Department of Pure Mathematics. His current research is Ordinary -partial differential equations, Graph Theory and Abstract Algebra.

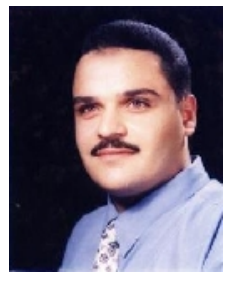

